Research Article

\title{
HIV as a prognostic factor for tuberculosis relapse: a systematic review and meta-analysis
}

\begin{abstract}
Aim: The systematic review objective is determine the magnitude of the association between HIV infection and TB relapse.

Background: Relapse refers to tuberculosis (TB) cases that have been treated and declared cured, or that has received complete treatment and is once again diagnosed with an episode of TB.

Materials and methods: Cohort-type studies that applied one or more genotyping methods and characterized the serological HIV status of patients were selected. Medline, Embase, BVS, and SciELO databases were used, along with Google and Google Scholar search engines, electronic journals in the Sinab network, grey literature, and bibliographical references from selected studies. Meta-analyses of subgroups were constructed according to the genotyping method, TB prevalence, and relapse definition. Risk of bias was evaluated and a sensitivity analysis was performed. OR estimators were established with and without studies that contributed the highest levels of heterogeneity.
\end{abstract}

Results: Eleven studies with 8941 patients were included. HIV (+) patients show 1.8 times greater risk than HIV (-) patients of presenting global relapse OR 1.78 [1.34, 2.38]. Regarding endogenous relapse, HIV is influential only in low prevalence zones OR 2.09 $[1.03,4.27]$, while for exogenous relapse, it is influential in high prevalence zones OR 4.42 $[1.56,12.57]$.

Conclusion: HIV is a prognostic factor for TB relapse. This relation is influenced by prevalence.

Keywords: Tuberculosis, Recurrence, HIV, Genotyping techniques, Polymorphism restriction fragment length, RFLP, VNTR sequences, Spoligotyping

Volume 4 Issue 3 - 2016

\author{
Monica Caicedo Roa,' Hernando G Gaitan \\ Duarte,' Martha Murcia Aranguren² \\ 'Faculty of Medicine, Universidad Nacional de Colombia sede \\ Bogot \\ ${ }^{2}$ Department of Microbiology, Universidad Nacional de \\ Colombia sede Bogot
}

\begin{abstract}
Correspondence: Monica Caicedo Roa, Universidad Nacional de Colombia sede Bogotá, Rua Carolina Germano Kokol II6 Vila Santa Isabel, Cundinamarca, Colombia, Campinas, São Paulo Brazil, CEP I 3084600 , Tel +55 (I I) 944537296, +57 (I) 3012886356, Email moincacaicedoroa@gmail.com
\end{abstract}

Received: November 28, 2016 | Published: December 28, 2016

\section{Secondary objectives}

\section{Precision of terms}

This review uses four terms to refer to the presentation of new tuberculosis episodes once the initial disease has cured. Recurrence is used in reference to a second episode of tuberculosis without specifying the mechanism through which it is presented. Relapse refers to the presentation of a second tuberculosis episode at a global level and is also used to refer to second episodes of the disease through an endogenous pathway. Reactivation is used specifically in reference to an endogenous relapse. Lastly, reinfection is only used in relation to the exogenous mechanism.

\section{Background}

\section{Tb Relapse}

The term relapse refers to tuberculosis cases that have been treated and declared cured, or that have received complete treatment and are once again diagnosed with an episode of TB, regardless of this being a true relapse or a new episode caused by reinfection. ${ }^{1}$ Depending on the relapse mechanism, two main forms are known: reinfection or infection through an exogenous pathway with a new Mycobacterium tuberculosis genotype, and reactivation through an endogenous mechanism with the same genotype that had been treated.

Genotyping by molecular biology techniques can be used to differentiate between the two relapse mechanisms. There are three main methods: Spoligotyping, ${ }^{2}$ MIRU VNTR, ${ }^{3}$ and RFLP-IS6110. ${ }^{4}$

\section{Objectives}

To determine the magnitude of the association between HIV infection and tuberculosis relapse through consolidation and evaluation of available evidence. a. To discriminate between tuberculosis relapse mechanisms based on endogenous or exogenous pathways through three genotyping methods in patients with and without HIV infection.

b. To evaluate the association based on local TB prevalence and treatment scheme.

\section{Methods}

Study types: Observational cohort-type studies that determined relapse diagnosis through one the the above mentioned genotyping techniques or a combination of these, and, where possible, an identification of the patient's serological state.

Participant type: Patients with a background of cured tuberculosis through combined treatment, regardless of age.

Exposure type: Infection with human immunodeficiency virus in carrier state, and stage 1 to 3 infection according to CDC. ${ }^{5}$

\section{Result types:}

Primary results: Global tuberculosis relapse and relapse through reinfection or reactivation mechanisms.

Secondary results: Resistance to Antituberculosis drugs, mortality, time until event (relapse), multiple relapse episodes.

Search methods for study identification: MeSH terms, Entree, and free language were used, along with truncators, Boolean connectors, orthographic variations, and abbreviations. No language types or year restrictions were used. 
Electronic databases search: The search strategy design process was supported by the coordinator of the Cochrane Group of Sexually Transmitted Infections of the Universidad Nacional de Colombia. Highly sensitive search strategies were used. MEDLINE ${ }^{\circ}$, EMBASE®, Google and Google Scholar, Bireme/ BVS, Science Direct, the first 1000 titles were included for this search, Electronic journals of the Sinab network related to tuberculosis.

Searches in other sources: Grey literature studies were obtained from relevant Open $\mathrm{Rey}^{6}$ electronic journals and databases. Congress abstracts related to tuberculosis and relapse were consulted, and bibliographical references were searched in the selected studies.

\section{Data collection and analysis}

\section{Selection of studies, Data extraction and management}

Two revisors independently reading titles and abstracts, and discrepancies were resolved through consensus, they extracted data through a standardized format. The data was compared and discrepancies were resolved by a collective review of the articles.

\section{Bias risk evaluation in the included studies}

The evaluation was done independently by two revisors, and the following tools were applied: Minors Methodological Index for NonRandomized Studies ${ }^{7}$ and Quality appraisal in systematic reviews of prognosis studies. $^{8}$

\section{Measurement of the effect}

Dicotomic data: The odds ratio, with IC 95\%, was used for dicotomic data presentation. Data was processed with the programs Review Manager $5.3^{9}$ and RStudio. ${ }^{10}$

Continuous data: time until event Relapse rates between exposed and non-exposed groups were compared; hazard ratios were estimated with confidence intervals of $95 \%$.

\section{Heterogeneity evaluation}

Study heterogeneity evaluation was done by elaborating a forest plot for global relapse and relapse by one of the two mechanisms. Additionally, the I2 statistic was calculated, using the inverse variance method, in order to determine study heterogeneity and to consider performing a meta-analysis through a random-effects model. To calculate T2, the maximum likelihood method was used. ${ }^{11}$ Furthermore, a Galbraith plot was done in order to identify the studies that contributed the greatest heterogeneity, and florest plot were constructed with and without the studies that contributed heterogeneity.

\section{Evaluation of publication bias}

A funnel plot was done and an Egger's test was applied.

\section{Analysis by subgroups}

a. According to the genotyping method employed.

b. Tuberculosis prevalence (low $<100$ cases $/ 100.000$ inhab. - high $>100$ cases/100.000 inhab.).

c. Relapse mechanism

\section{Sensitivity analysis}

A global estimation, in addition to an estimation with only low bias risk-studies according to each of the evaluation methods, was done in order to identify variations in the OR estimator due to the methodological quality of the studies.

\section{Ethical considerations}

The research question and protocol to answer it consider the ethical principles of health research and investigation norms. The research protocol was approved by the ethics committee of the Facultad de Medicina of the Universidad Nacional, although it was not disclosed on the web.

\section{Results}

\section{Search results}

The search was performed on the referenced databases and other resources, by applying search strategies. 14 studies were selected ${ }^{12-24}$ (Table 1), including 11 that contributed complete data, and three additional studies which did not provide information Caminero, Narayanan \& Cacho et al. ${ }^{20,24,25}$ Electronic correspondence was mailed to the authors requesting the missing information. A response was obtained from two of them, who claimed to not have access to the missing information (See Figure 1).

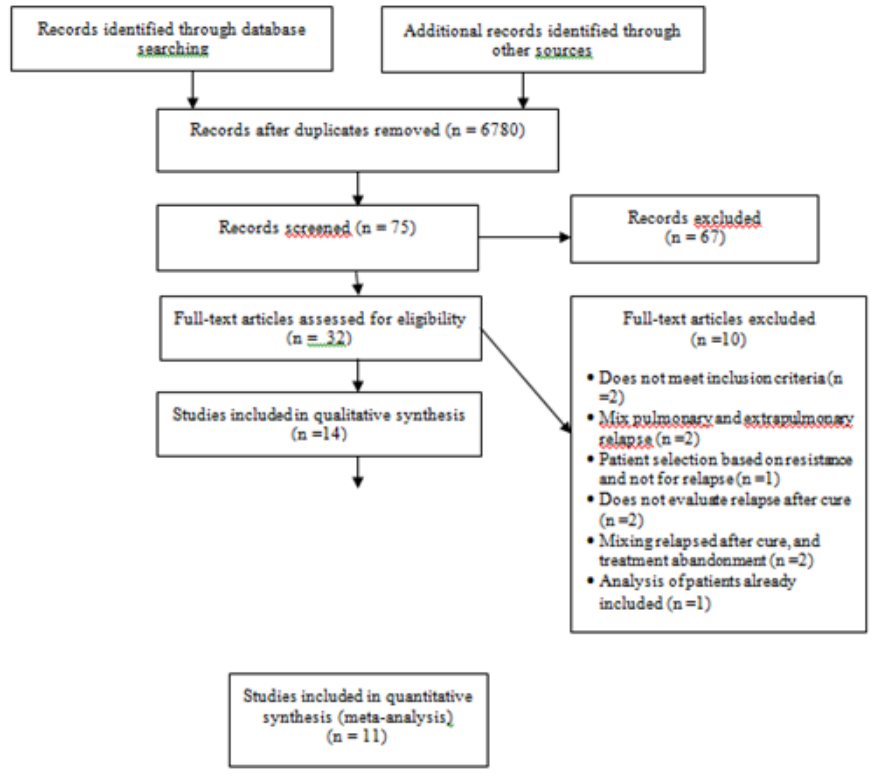

Figure I Flow chart of included studies.

\section{Included studies}

\section{General characteristics}

Table 1 shows a description of the main data from the studies. The 14 cohorts represent diverse populations: from localized groups with high risk, such as miners, to other more generalized groups taken from national vigilance systems; a third group was centered on hospitals or clinics, and the latter of these was composed of follow-up cohorts assembled after finalizing clinical trials. All of these cohorts included adult patients with cured tuberculosis by combined treatment.

\section{Global relapse and relapse mechanisms}

Among the 14 included studies, 11 provided complete data of the number of patients with and without HIV infection and also of the relapse serological states. These studies included 8941 patients, of which $45 \%(n=4.002)$ were HIV positive. In general, 512 relapses were identified (5.7\% of risk patients), 309 in positive patients and 203 in negative patients. This is proportionally equivalent to $8 \%$ and $4 \%$ relapse per HIV group. 


\section{Heterogeneity sources}

Various heterogeneity sources were found in the review. Visually, confidence intervals in the forest plot overlap between studies Figure 2. Heterogeneity is considerable, ranging from $75 \%-100 \%$ for general and significant relapse, and 50\%-90\% for genotyping mechanisms. ${ }^{26}$

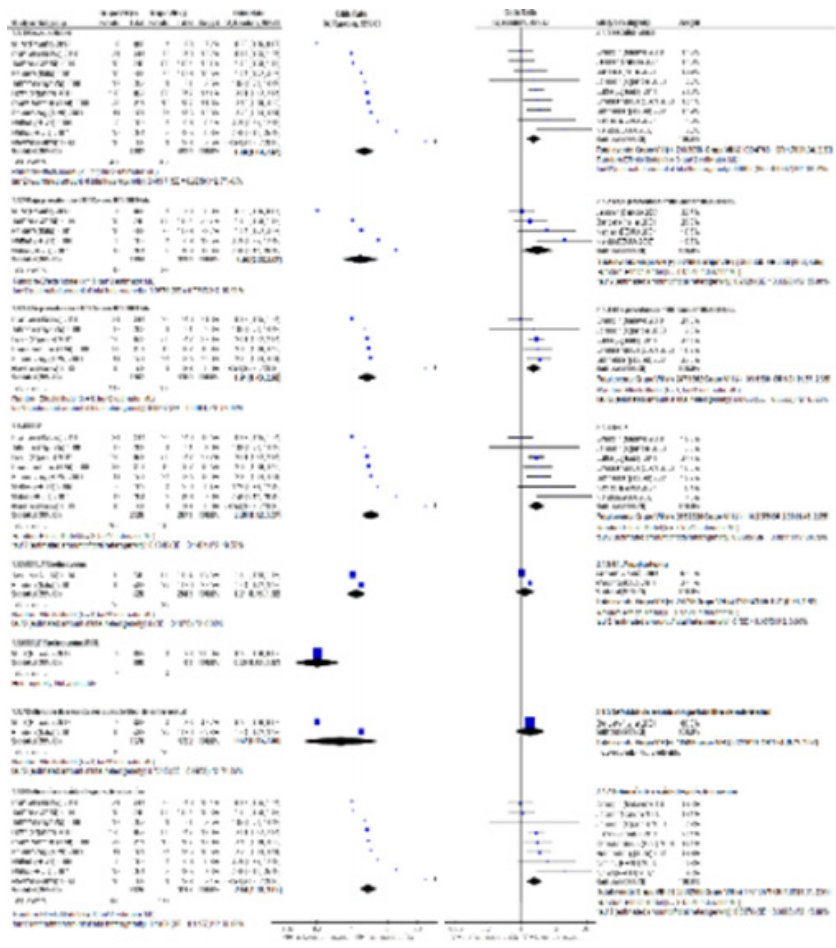

Figure 2 Left Forest plot: comparison of global relapse of HIV (+) and HIV (-) patients; Right Forest plot: comparison of global relapse of HIV (+) and HIV (-) patients without Hawken \& Millet et al. ${ }^{15,23}$

The Galbraith plot shows the two studies that contribute the most heterogeneity, Hawken \& Millet et al. ${ }^{15,23}$ therefore, tree diagrams with and without these studies were constructed.

\section{Global relapse}

Global relapse events include relapses without discriminating by mechanism. Globally, patients with HIV infection are estimated to have twice as much risk of presenting relapse compared to negative patients OR $1.80[1.09,2.97]$ Figure 2 analysis 1.1.1. The analysis by high and low prevalence subgroups Figure 2 right analyses 1.1.2 and 1.1.3 shows that the estimated risk decreases in low tuberculosis prevalence zones OR $1.46[0.55,3.87]$. In high prevalence zones, with $>100$ cases $/ 100.000$ inhabitants, the effect persists OR 1.91 $[1.40,2.60]$ in a statistically significant manner. For these estimators, heterogeneity (I2) was of $77 \%, 81 \%$, and $24 \%$.

When the studies contributing heterogeneity ${ }^{15,23}$ are removed, Figure 2 left is obtained, which shows how the estimations for global relapse persist, with lower heterogeneity levels, for global relapse events OR 1.78 [1.34, 2.38] Figure 2 analysis 2.1.1. After discriminating by high and low prevalence zones, HIV is shown as an influential factor in tuberculosis relapse 2.03 and 1.81, respectively, Figure 2 analyses 2.1 .2 and 2.1.3, (I2) of $34 \%, 56 \%$, and $15 \%$.

\section{Genotyping methods for global relapse}

Among cohorts, nine used RFLP individually, two used RFLP combined with Spoligotyping, and one with MIRUS. Only two studies used a combination of the three methods: Narayanan \& Millet et al. ${ }^{20,23}$ The global relapse subgroups analysis shows a stratified analysis by each method for 11 studies with complete data Figure 2 (analyses 1.1.4, 5, and 6).

Controlling for the RFLP genotyping method, it is observed that HIV continues to be an influential factor in relapse development OR 2.26 [1.52, 3.37]; the estimator calculated based on data from eight cohorts with a heterogeneity of $49 \%$.

For the RFLP/Spoligotyping group, an OR of 1.021 is obtained, with a non-significant confidence interval of $95 \%[0.76,1.92]$. This group is made up of two studies, both with different relapse definitions. Lastly, the third stratum includes RFLP/Spoligotyping/ MIRU, in which only one study provided complete relapse data and shows HIV as a protective factor 0.20 [0.06, 0.67] for relapse, I2 57\%.

After removing the studies that contributed heterogeneity, the estimations are mantained with acceptable levels $(29 \%$ and $0 \%)$ for RFLP/Spoligotyping, and the protective effect identified by Miller et al. ${ }^{23}$ dissappears.

\section{Definition for global relapse}

A third definition-dependent stratified analysis was performed Figure 2 (analyses 1.1.7 and 8). Although this analysis was not included in the protocol, it was considered relevant due to differences observed between three studies regarding the relapse concept in tuberculosis disease.

For the remaining studies, relapse was considered from the moment of being cured Figure 2 (analysis 1.1.8). This relapse was presented in $11 \%$ of positive patients and $5.5 \%$ of negative patients, with an OR of $2.04[1.38,3.02]$ and a heterogeneity of $57 \%$.

After removing the studies that contributed the most heterogeneity, it is observed that for definitions with and without a disease-free period, HIV is a prognostic factor for relapse Figure 2 right (analyses 2.1.6 and 7) with I2 43\%, OR of $1.54[0.72,2.27]$ and 1.83 [1.31, $2.55]$, respectively.

\section{Relapse by an endogenous mechanism}

Globally, 115 events among HIV (+) patients and 148 among HIV (-) patients were obtained. Figure 3 (analysis 1.2.1) shows that relapse by this mechanism has an OR of 1.22 IC $95 \%$ [0.75, 1.97] I2 55\%, and, after stratifying for prevalence, this estimator is non-conclusive. High prevalence OR of $0.88[0.61,1.25]$ I $20 \%$, and low prevalence OR $1.78[0.86,3.69]$ I2 55\% Figure 3 (analyses 1.2.2 and 3).

After excluding heterogenous studies from the analysis, the estimations are mantained and, in the general analysis, endogenous relapse is significant in low tuberculosis prevalence zones OR of 2.09 [1.03, 4.27], I2 56\%. This does not occur in high prevalence zones, where endogenous relapse seems to not be influenced by HIV infection OR of $0.85[0.56,1.21]$ Figure 3 right.

\section{Relapse by an exogenous mechanism}

Eight of eleven studies were included, since three of them did not present events by this mechanism. According to the general analysis, an OR of 2.25 [0.67, 7.52], I2 63\% was obtained. Exogenous relapses are less frequent compared to endogenous relapses; proportionally, these were present in $1.4 \%$ of the HIV $(+)$ group and in $0.42 \%$ of the HIV (-) group. By stratifying the results according to prevalence Figure 4 (analyses 1.3.2 and 3), it is observed that these events are less frequent in low prevalence zones, with two and six cases OR of 0.34 $[0.34,3.47]$, I $260 \%$. In contrast, in high prevalence zones, HIV is a 
significant factor for relapse by an exogenous mechanism OR of 4.58 $[1.70,12.12]$ I2 29\%.

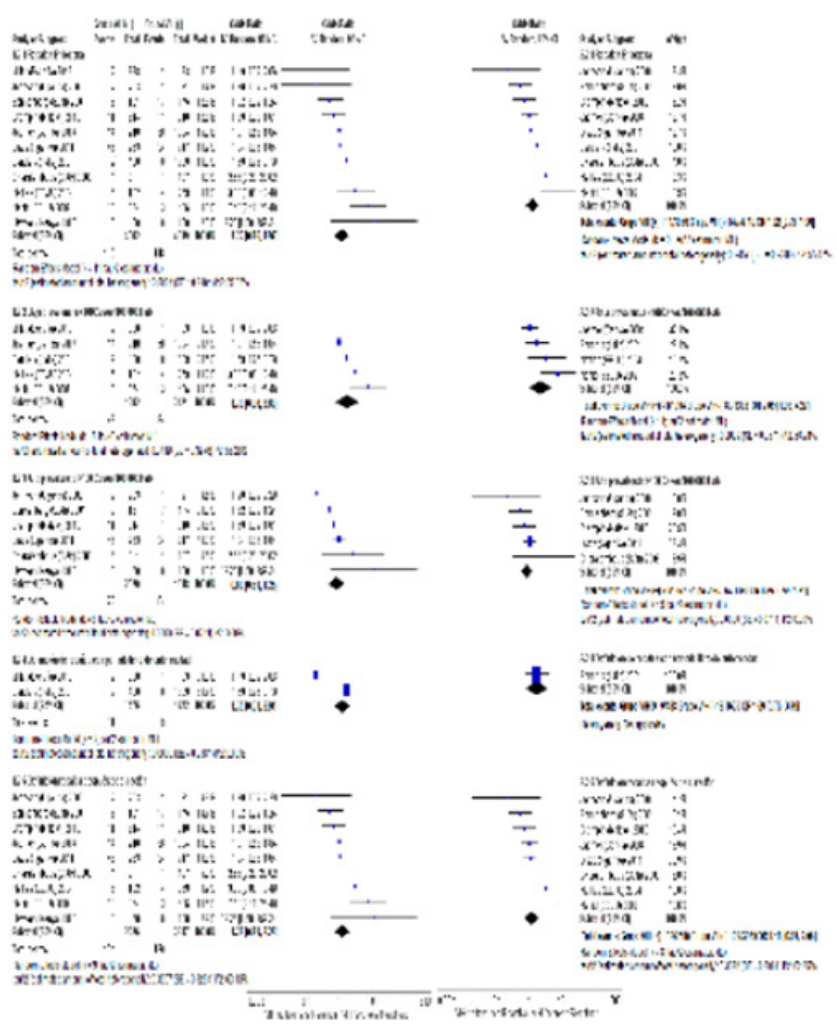

Figure 3 Left Forest plot: comparison of endogenous relapse of HIV (+) and HIV (-) patients; Right Forest plot: comparison of endogenous relapse of HIV $(+)$ and HIV (-) patients without Hawken 1993 and Millet 2013.

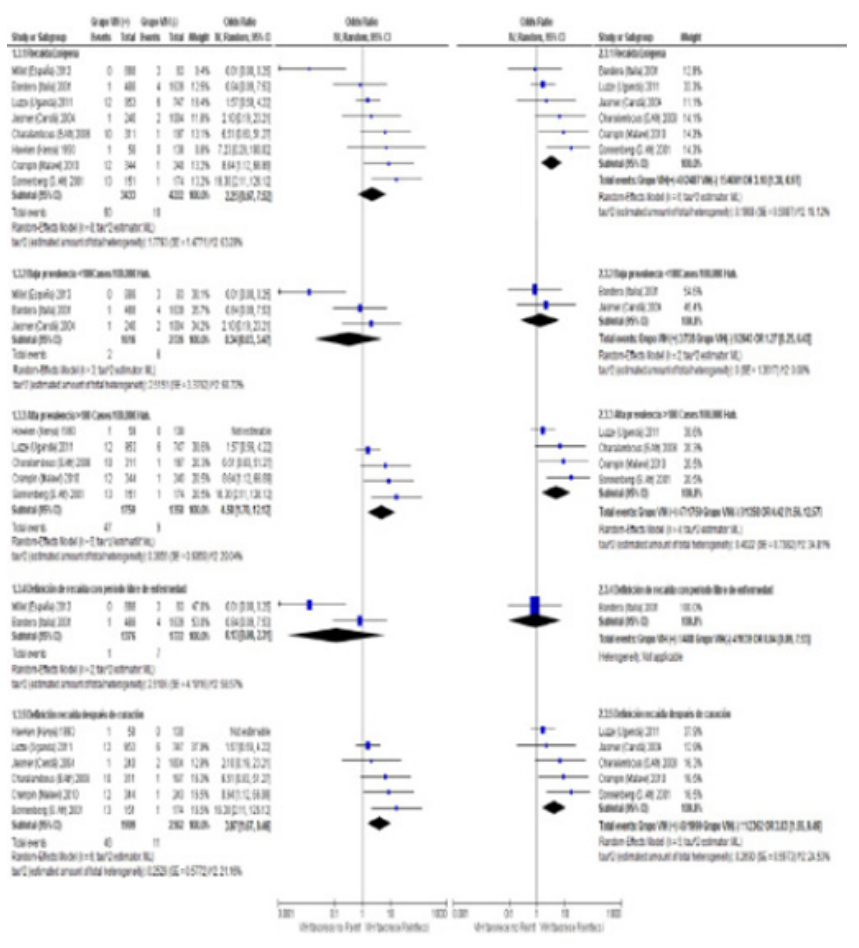

Figure 4 Left Forest plot: comparison of exogenous relapse of HIV (+) and HIV (-) patients; Right Forest plot: comparison of exogenous relapse of HIV $(+)$ and HIV (-) patients without Hawken \& Millet et al..$^{15,23}$
After removing studies that contributed the greatest heterogeneity Figure 4 right, global estimation for exogenous relapse improves with an OR of $3.10[1.38,6.97] \mathrm{I} 218 \%$, and it becomes more influential in the high prevalence subgroup OR of $4.42[1.56,12.5]$.

\section{Time until Event}

The results vary among studies, from the lowest: Jasmer (16) HR of 1.10 [0.96, 1.27], to the highest: Hawken et al. ${ }^{15} \mathrm{HR}$ of 33.40 $[14.03,104.2]$. It is worth mentioning that the latter study used an initial scheme with thioacetazone and patients had to be migrated to regimes with rifampicin due to presenting hypersensitive reactions, which indirectly led to these patients presenting lower treatment adherence indices. Among studies, six HR estimations are significant; in this event, data were not clustered, since I 2 was close to $98 \%$.

Among the remaining studies that showed statistically significant HR, all are above 2.4; thus, positive patients can be expected to present relapse in half the observation time compared to the negative group.

\section{Resistance}

Seven studies did not provide insight on bacilli sensitivity during relapse, compared to the initial sensitivity profiles. Sixteen resistance forms could be identified in HIV positive patients, of which seven were towards rifampicin, four to isoniazid, one to ethambutol, and one to streptomycin. Only one multiresistance was observed, in Nahid et al. ${ }^{21}$ and one polyresistance, in Bandera et al. ${ }^{12}$ the latter with the greatest sample size among cohorts.

Regarding the HIV negative group, six resistance profiles were identified, two of them towards rifampicin, two to isoniazid, one to streptomycin, and a multiresistance.

\section{Mortality}

Mortality was poorly evaluated among studies. Eight of them did not provide data; only Crampin et al. ${ }^{14}$ mentioned mortalities in the group and among patients with relapse: 180/9 in HIV (+) and 15/2 in HIV (-), respectively. The remaining studies reported mortalities in the general group, although not recurrence-associated. In total, 465 positive patients and 65 negative patients.

\section{Multiple recurrence episodes and HIV staging}

The included studies did not evaluate multiple recurrence episodes and did not provide HIV stages.

\section{Bias risk of the included studies}

Two bias risk evaluation tools were used and the results are as follows:

Minors: This tool provides a grading scale of: 0 for not reported (-), 1 for reported but inadequate (?), and 2 reported and adequate $(+)$ (Figure 5).

Quality Appraisal in Systematic Reviews of Prognosis Studies: For the second tool, six categories were evaluated based on an ordinal grading scale: yes (+), partially/unsure (?), and no (-) (Figure 5).

\section{Publication bias}

Publication bias was estimated based on the funnel plot in Figure 6, which shows symmetry. Additionally, an assymetry regression Egger test was applied (Regression: Asymmetry: $\mathrm{z}=1.3565) \mathrm{p}=0.1749$, which indicated no publication bias. 


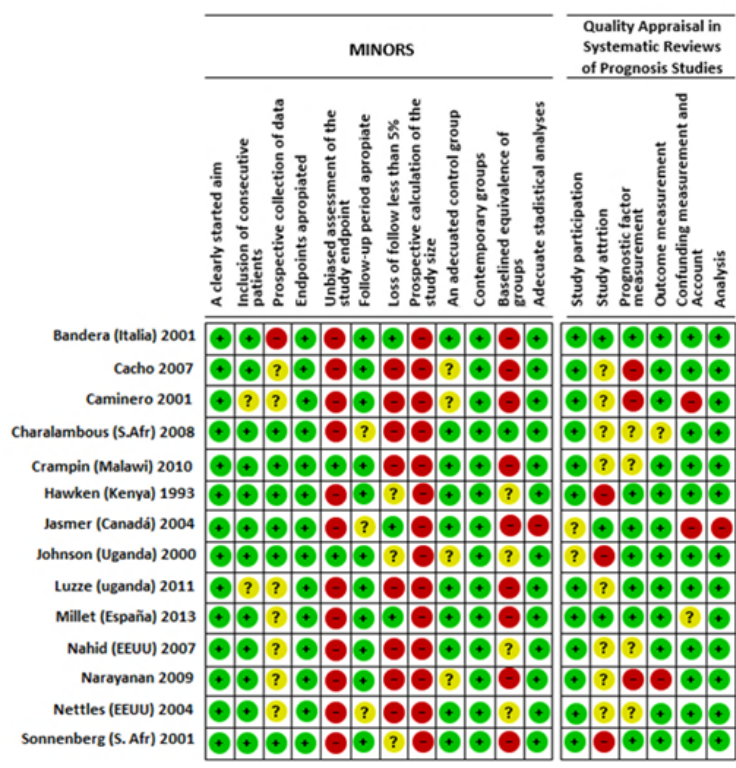

Figure 5 Bias risk based on the Minors and Quality Appraisal in Systematic Reviews of Prognosis Studies bias evaluation tools, discriminated by study.

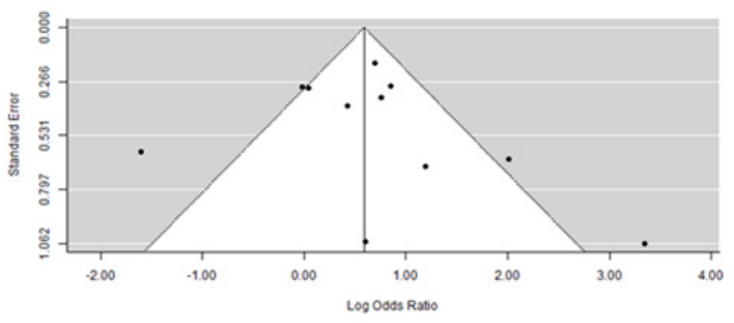

Figure 6 Funnel plot

\section{Sensitivity analysis by bias risk}

After recognizing the studies limitations through the two bias risk evaluation methods, new forest plots were constructed in order to analyze OR variation when only low risk studies were included.

By applying this procedure, the results from Table 2 were obtained, which show the variation for each of the events studied.

The tool that most contributes to variation in the OR estimation is Minors. Based on this tool, global relapse loses estimation significance from 0.63 to 4.22 , with a confidence interval of $95 \%$, and endogenous relapse shifts in estimation direction from an OR of 1.22 to 0.64 . No important variations are observed for exogenous relapse; only a decrease in precision is observed, based on a broader confidence interval

According to Minors evaluation, the greatest variation is shown in the amplitude of the confidence intervals, which can explain the reduction in the number of patients in the comparison groups.

\section{Discussion}

\section{Synthesis of the main results}

The main result of this systematic review allows answering the research question and defining whether HIV is a prognostic factor of tuberculosis relapse. The conclusion can be obtained from the general event, which includes the total relapses; the clustered estimator,
OR, was of 1.78 [1.34, 2.38], I2 of $34 \%$. This effect is significantly persistent in high prevalence zones: OR of 1.81 [1.37, 2.39], as well as when the RFLP method is used individually.

The OR estimator loses significance in the combined genotyping methods subgroup (RFLP/Spoligotyping), since the sample size is not enough to calculate an estimator with a significant confidence interval; in other words, it does not have enough statistical power to identify the differences between HIV-exposed and not exposed groups. Specific diagnostic studies are necessary in order to evaluate the effect of individual or combined genotyping methods for relapse detection.

Eight percent of patients with co-infection suffer relapse; the main relapse mechanism is endogenous (37\%) and, in a lesser proportion, exogenous (16\%). Four percent of patients in the control group present global recurrence; of these, $73 \%$ suffer relapse through an endogenous pathway.

After removing studies that contributed heterogeneity, the meta-analysis shows the influence of HIV in endogenous relapse development OR of $1.22[0.76,1.96]$ and its importance in low prevalence zones, where it is the main relapse mechanism OR of 2.09 $[1.03,4.27]$. On the other hand, the meta-analysis shows that HIV affects reinfection (exogenous mechanism) OR of 3.10 [1.38, 6.97] and its influence is persistent in high prevalence zones OR of 4.42 $[1.56,12.57]$. This means that in environments with low tuberculosis prevalence, relapse of patients with HIV occurs by an endogenous mechanism, while, in high prevalence locations, the main relapse mechanism is through reinfection. This is biologically plausible, given that, in high prevalence zones, there is a greater probability of reinfection.

A stratified analysis of the studies according to their relapse definition was performed. In studies where relapse is defined after the tuberculosis infection is cured, HIV is observed as a definitive factor in the presentation of the event OR of 1.83 [1.31, 2.55]. On the contrary, in studies where relapse is established after a tuberculosis-free time period (up to one year), the effect of HIV is diluted. Given that most relapses $(90 \%)$ occur during the first year after tuberculosis is cured, the second definition would not allow to observe most episodes. Millet et al. ${ }^{23}$ reported nine and four relapses in HIV (+) and HIV (-) patient groups, equivalent to $1 \%$ and $5 \%$, respectively. As can be observed, most relapses take place in HIV (-) patients, which usually take longer to develop a relapse or present a reinfection episode.

One of the main difficulties identified in the cohorts was the lack of availability of the genotyping results that would allow a recurrence sub classification. These results were available in only $65 \%$ of relapses.

Regarding event timing, all studies agreed that relapse occurs earlier in patients with co-infection. However, there is a broad variation between $\mathrm{HR}$ estimated among the studies, from 1.10 [0.96, 1.27] to 33.40 [14.03, 104.2], which indicates that post-treatment follow-up of this patient group must be done more exhaustively.

These estimations must be interpreted according to the methodological quality of the studies, since there is a considerable bias risk observed in this review for selection, measurement, and differential loss. However, in the sensitivity analysis, after removing studies qualified as high risk according to the Minors questionnaire, only OR variations were obtained in relation to endogenous relapse, given that there was a shift in the effect direction from 1.22 to 0.64 . In relation to the second tool (global relapse, endogenous relaspe, and exogenous relapse events), the OR persist. 


\section{Limitations}

One of the obstacles found during the realization of this review was the high variability among treatments in terms of duration, frequency, and drugs administered. Furthermore, the authors do not mention the effectiveness of these treatments in the study locations, and many of them do not indicate cure rates.

It is also worth noting that in only six of fourteen studies, the treatment was administered under direct observation conditions. A strict supervised administration affects treatment effectiveness by improving adherence and favoring sputum conversion after two months. Treatments that are not administered under these conditions can affect resistance development and disease reactivation. Nevertheless, the data from this review do not allow to retrieve conclusions in this regard due to the restricted number of mono and multiresistances detected.

The treatment used for pulmonary tuberculosis affects relapse development. However, given treatment variability among studies, it was not possible to evaluate this influence through an adjusted analysis.

\section{Heterogeneity}

The studies show various sources of heterogeneity, both clinical and methodological. Regarding clinics, differences among patient characteristics, health conditions, treatments used, and exposure risk were observed.

In relation to the methodological heterogeneity, sample sizes present a marked variation, with a median of 596 patients, range 196 -2127 patients. Observational studies are more prone to presenting unbalances in patient characteristics.

Other sources of methodological heterogeneity are determined by prospective and retrospective cohort designs, relapse definitions used by the authors, durations of the study periods (mean follow-up times were 7.05 years SD 4.3 years), and variations among treatment schemes, which differed in all of the studies and are unknown in four of them Table 1. A way of quantifying the effectiveness of the treatments used consisted of establishing the cure rate, which was specified in only six studies and, in general, was above $70 \%$, with the exception of Millet 2013, which was of $65 \%$.

Despite the differences among studies, adjusted meta-analyses were performed, eliminating those that contributed the greatest levels of heterogeneity. This allowed to obtain clustered estimators. These estimators proved to be stable, with acceptable I2 levels.

\section{Potential bias in the review process}

This review was done according to the guidelines of the "Cochrane Handbook for Systematic Reviews of Interventions"26 and counted on the supervision and approval from topic and methodology experts. We expect that the information was exhaustive enough to identify all of the studies that met the inclusion criteria. The bias risk evaluation was done according to two methods in order to clearly identify the studies' limitations.

No clinical trials evaluating tuberculosis relapse were found; therefore, the review was done based on observational studies.

Heterogeneity among studies is important. For this reason, heterogeneity is explicitly reported in each one of the estimators used and a description of its possible clinical and methodological sources is given. In addition, independent meta-analyses are elaborated with and without the studies that contributed the greatest heterogeneity in order to evaluate the behavior of the clustered OR and its confidence interval.

\section{Agreements and disagreements with other studies and reviews}

Three previous systematic reviews were found: Lambert, Korenromp \& Panjabi et al. ${ }^{27-29}$ which sought to determine HIV importance in tuberculosis recurrence; as well as a narrative review: Schiroli et al. ${ }^{30}$

The present review and the one by Korenromp et al. ${ }^{28}$ differ in that the author initially restricted cohorts to those that used rifampicinbased tuberculosis treatments in the first and second phases. However, given that this restriction limited the number of studies, Korenromp also included retrospective cohorts and treatments without rifampicin, with which he completed 47 works, 21 of these included HIV (+) patients and 41 included HIV (-) patients. The discrepancy was due to the fact that the author included cohorts from only one group. This was not considered in this review due to the difficulty in comparing relapse events in HIV-exposed and non-exposed patients from different populations.

In the three reviews, the bias risk was not evaluated and the systematic search was done mainly in two databases (the most frequent was Medline). Lambert et al. ${ }^{27}$ and Korenromp et al. ${ }^{28}$ mention differences in relapse concepts, among the limitations of their reviews, which complicate result interpretations. In this review, this limitation was addressed through a stratified analysis which allowed to identify that the relapse concept affects the association between tuberculosis relapse and HIV infection.

Korenromp et al. ${ }^{28}$ highlights a possible selection bias derived from limiting the studies to those with positive sputum culture. This can lead to underestimating the effect of HIV infection, since AIDS is associated with negative culture results. Korenromp also mentions that some studies do not report loss of individuals, while other studies reach a rate of up to $20 \%$, a deficiency that was also evaluated in this review.

Korenromp et al. ${ }^{28}$ reported recurrence rates of $7 \%$ in HIV $(+)$ and $4 \%$ in HIV (-), values similar to those found in this review.

Lambert et al. ${ }^{27}$ found high heterogeneity levels among studies and deficiencies in drug resistance reports. Both problems were also observed in this review. To address the former, clustered estimators were calculated based on meta-analyses with and without studies that contributed the greatest heterogeneity. Addressing the second problem (lack of data) is not possible. Regarding this lack of information, it is necessary for primary studies to provide sensitivity profiles in the first and second tuberculosis episodes in order to identify resistance development.

Panjabi et al. ${ }^{29}$ included 32 studies, 18 clinical trials and 14 observational studies, of which only four included genotyping, and mentioned that recurrence rates were greater in observational studies than in experimental studies. Based on this indication, and given the advantages that observational studies offer in terms of follow-up time and approximation to real treatment conditions among the population, we consider that these studies constitute the best methodological design for evaluating relapse. Panjabi's review found 20 studies in Medline and 12 through bibliographical references and expert opinions. Our review also found a considerable number of additional cohorts among bibliographical references in the elegible studies. This search strategy limits the risk of not identifying studies of interest; thus, it should be considered indispensable in elaborating secondary studies. 


\section{Conclusions and authors recommendations}

i. HIV favors the occurrence of subsequent tuberculosis episodes, both through endogenous and exogenous mechanisms. This relation is influenced by prevalence. Another factor that can affect the relation between HIV and tuberculosis relapse is treatment. However, this review does not contain enough evidence to establish an effect by treatment, due to the high variation found in schemes, regarding duration, drug combination in the first and second phases, effectiveness, and proportion of patients that received strict supervised treatment.

ii. HIV is a prognostic factor of tuberculosis recurrence in terms of global relapse and endogenous relapse, since it affects the presentation of new tuberculosis episodes after presenting the initial disease. Endogenous relapses have even been regarded as a continuation of the original tuberculosis disease. On the other hand, in relation to relapse by an exogenous mechanism, it would be appropriate to refer to HIV as a risk factor, and not as a prognostic factor, since this mechanism implies that the initial disease was cured and a presentation of a totally different episode confirmed based on genotyping.

iii. Observational cohort-type studies are the best methodological design for investigating the effect of HIV exposure on tuberculosis relapse. Genotyping methods allow to reliably discriminate between relapse mechanisms. However, a guaranteed availability of Mycobacterium tuberculosis cultures taken in the initial and second episode is necessary in order to compare DNA sequences. Other approaches, such as resistance profiles, are not equally valid for determining the relapse mechanism and could bias the results towards the endogenous pathway.

iv. Complementary search methods, such as the review of bibliographical references in eligible studies, are a good strategy for finding additional potentially useful studies, which reduces the publication bias risk

v. The analysis of Galbraith plots allows to identify studies that contribute the greatest heterogeneity. This allows, in turn, to evaluate shifts in OR estimation through the construction of a meta-analysis with and without these studies. The meta-analysis is considered valid if, once the most heterogenous studies are removed, the OR value and its confidence interval do not lose statistical significance and if, in addition, the heterogeneity can be reduced to acceptable levels.

vi. Authors of primary studies must provide comparison group characteristics in order to identify differences than can modify research results. Moreover, authors should mention the schemes and conditions of the administered treatments.

\section{Differences between the protocol and the review}

The temporal restriction proposed in the protocol (studies published since 1995) was removed. This limitation was intended to include studies with controlled treatments; however, since the studies did not present directly observed treatments, we decided to broaden the search.

The heterogeneity tolerance range was broadened $(\geq 40 \%)$ to perform the meta-analysis. Likewise, we decided to report the I2 of the main results and construct tree diagrams with and without the studies that contributed the greatest heterogeneity.
In the search methodology for potential studies, the snowball search strategy was replaced by the review of references from eligible studies.

After finding different relapse definitions in the studies, an analysis by subgroups was performed for those studies that established disease-free periods of 6 and 12 months, which was not included in the protocol.

\section{Summary}

HIV is a prognostic factor for TB relapse. HIV (+) patients show 1.8 times the risk HIV (-) of presenting global relapse. For endogenous relapse, HIV is influential only in low prevalence zone, while for exogenous relapse, it is more influential high prevalence.

\section{Acknowledgments}

To the Universidad Nacional de Colombia for providing the resources for the realization of this systematic review through the Grant: Programa Nacional de Proyectos para el Fortalecimiento de la Investigación, la Creación y la Innovación en Posgrados 20132015 modalidad 2 (project number 18984). To the statistical advisory program for postgraduate research of the Universidad Nacional de Colombia.

\section{Conflicts of interest}

None.

\section{References}

1. World Health Organization(WHO). Definitions and reporting framework for tuberculosis. Geneva. 2013.

2. Kamerbeek J, Schouls L, Kolk A, et al. Simultaneous detection and strain differentiation of Mycobacterium tuberculosis for diagnosis and epidemiology. J Clin Microbiol. 1997;35(4):907-914.

3. Supply P, Lesjean S, Savine E, et al. Automated high-throughput genotyping for study of global epidemiology of Mycobacterium tuberculosis based on mycobacterial interspersed repetitive units. J Clin Microbiol. 2001;39(10):3563-35671.

4. van Embden JD, Cave MD, Crawford JT, et al. Strain identification of Mycobacterium tuberculosis by DNA fingerprinting: recommendations for a standardized methodology. J Clin Microbiol. 1993;31(2):406-409.

5. Schneider E, Whitmore S, Glynn K, et al. Revised Surveillance Case Definitions for HIV Infection Among Adults, Adolescents, and Children Aged $<18$ Months and for HIV Infection and AIDS Among Children Aged 18 Months to <13 Years_— United States, 2008;57(RR10):1-8.

6. System for Information on Grey Literature in Europe. CNRS- Centre national de la recherche scientifique, France.

7. Slim K, Nini E, Forestier D, et al. Methodological index for nonrandomized studies (minors): development and validation of a new instrument. ANZ J Surg. 2003;73(9):712-716.

8. Hayden JA, Cote P, Bombardier C. Evaluation of the quality of prognosis studies in systematic reviews. Ann Intern Med. 2006;144(6):427-437.

9. Review Manager. Computer program. Version 5.3. Copenhagen: The Nordic Cochrane Centre, The Cochrane Collaboration, Denmark. 2014.

10. R Studio Team. R Studio: Integrated Development for R. R Studio, Inc., Boston, MA, USA. 2015.

11. Huedo T, Sanchez J, Marin F. La estimación del tamaño del efecto medio en un meta análisis: Una comparación entre los modelos de efectos fijos y aleatorios. 2003 
12. Bandera A, Gori A, Catozzi L, et al. Molecular epidemiology study of exogenous reinfection in an area with a low incidence of tuberculosis. $J$ Clin Microbiol. 2001;39(6):2213-2218.

13. Charalambous S, Grant AD, Moloi V, et al. Contribution of reinfection to recurrent tuberculosis in South African gold miners. Int J Tuberc Lung Dis. 2008;12(8):942-948.

14. Crampin AC, Mwaungulu JN, Mwaungulu FD, et al. Recurrent TB: relapse or reinfection? The effect of HIV in a general population cohort in Malawi. AIDS. 2010;24(3):417-426.

15. Hawken $M$, Nunn $P$, Gathua $S$, et al. Increased recurrence of tuberculosis in HIV-1-infected patients in Kenya. Lancet. 1993;342(8867):332-337.

16. Jasmer RM, Bozeman L, Schwartzman K, et al. Recurrent tuberculosis in the United States and Canada: relapse or reinfection? Am J Respir Crit Care Med. 2004;170(12):1360-1366.

17. Sonnenberg P, Murray J, Glynn JR, et al. HIV-1 and recurrence, relapse, and reinfection of tuberculosis after cure: a cohort study in South African mineworkers. Lancet. 2001;358(9294):1687-1693.

18. Johnson JL, Okwera A, Nsubuga P, et al. Efficacy of an unsupervised 8-month rifampicin-containing regimen for the treatment of pulmonary tuberculosis in HIV-infected adults. Uganda-Case Western Reserve University Research Collaboration. Int J Tuberc Lung Dis. 2000;4(11):1032-1040.

19. Luzze H, Johnson DF, Dickman K, et al. Relapse more common than reinfection in recurrent tuberculosis 1-2 years post treatment in urban Uganda. Int J Tuberc Lung Dis. 2013;17(3):361-367.

20. Narayanan S, Swaminathan S, Supply P, et al. Impact of HIV infection on the recurrence of tuberculosis in South India. J Infect Dis. 2010;201(5):691-703.

21. Nahid P, Gonzalez LC, Rudoy I, et al. Treatment outcomes of patients with HIV and tuberculosis. Am J Respir Crit Care Med. 2007;175(11):1199-1206.
22. Nettles RE, Mazo D, Alwood K, et al. Risk factors for relapse and acquired rifamycin resistance after directly observed tuberculosis treatment: a comparison by HIV serostatus and rifamycin use. Clin Infect Dis. 2004;38(5):731-736.

23. Millet JP, Shaw E, Orcau A, et al. Tuberculosis recurrence after completion treatment in a European city: reinfection or relapse? PLOS One. 2013;8(6):e64898.

24. Caminero JA, Pena MJ, Campos-Herrero MI, et al. Exogenous reinfection with tuberculosis on a European island with a moderate incidence of disease. Am J Respir Crit Care Med. 2001;163(3 Pt 1):717-720.

25. Cacho J, Pérez Meixeira A, Cano I, et al. Recurrent tuberculosis from 1992 to 2004 in a metropolitan area. Eur Respir J. 2007;30(2):333-337.

26. Higgins JPT, Green S. Cochrane Handbook for Systematic Reviews of Interventions. Version 5.1.0 [updated March 2011]. The Cochrane Collaboration, Handbook information. 2011.

27. Lambert ML, Hasker E, Van Deun A, et al. Recurrence in tuberculosis: relapse or reinfection? Lancet Infect Dis. 2003;3(5):282-287.

28. Korenromp EL, Scano F, Williams BG, et al. Effects of human immunodeficiency virus infection on recurrence of tuberculosis after rifampin-based treatment: an analytical review. Clin Infect Dis. 2003;37(1):101-112.

29. Panjabi R, Comstock GW, Golub JE. Recurrent tuberculosis and its risk factors: adequately treated patients are still at high risk. Int $J$ Tuberc Lung Dis. 2007;11(8):828-837.

30. Schiroli C, Franzetti F. Recurrent tuberculosis: relapse or reinfection? Infez Med. 2013;21(4):251-260. 